\title{
Tree nuts and the lipid profile: a review of clinical studies
}

\author{
Amy E. Griel and Penny M. Kris-Etherton* \\ The Pennsylvania State University, S-126 Henderson Building, University Park, PA 16802, USA
}

\begin{abstract}
Tree nuts have a fatty acid profile that favourably affects blood lipids and lipoproteins. They are low in saturated fat and high in unsaturated fatty acids and are rich sources of other nutrients. An extensive database consistently shows total and LDL cholesterol-lowering effects of diets low in saturated fat and cholesterol and high in unsaturated fat provided by a variety of tree nuts. Collectively, a summary of studies conducted to date shows that tree nuts reduce LDL cholesterol by 3-19\% compared with Western and lower-fat diets. Nuts also contain many nutrients and bioactive compounds that appear to contribute to the favourable effects on lipids and lipoproteins - these include plant sterols, dietary fibre and antioxidants. Because of their unique nutrient profile, nuts can be part of a diet that features multiple heart-healthy foods resulting in a cholesterol lowering response that surpasses that of cholesterol-lowering diets typically used to reduce CVD risk.
\end{abstract}

Tree nuts: Lipids: Lipoproteins: Clinical trials: Humans

Numerous clinical studies have been conducted to evaluate the effects of nut consumption on lipids and lipoproteins, major risk factors for cardiovascular disease (CVD) (reviewed by Kris-Etherton et al. 2001; Mukuddem-Petersen et al. 2005). The impetus for the clinical studies were the results of several major epidemiologic studies that have demonstrated beneficial effects of nut consumption on coronary disease risk (Fraser et al. 1992; Fraser \& Shavlik, 1997; Fraser et al. 1997; Hu et al. 1998; Hu \& Stampfer, 1999; Ellsworth et al. 2001; Albert et al. 2002; Jiang et al. 2002). Importantly, these results have been demonstrated consistently in all population groups studied, including men and women of all ages, African-Americans and persons with diabetes. The clinical database for nuts is increasing rapidly and the preponderance of evidence is on lipid and lipoprotein endpoints as a means to explain epidemiologic results.

The clinical studies have assessed the effects of different tree nuts, including walnuts, almonds, macadamia nuts, pecans, pistachios and hazelnuts. Many experimental designs have been employed with different population groups. For example, studies have been conducted with free-living subjects on selfselected diets who were given either specific instructions to follow regarding nut consumption or daily allotments of nuts to consume. Controlled feeding studies also have been employed in which subjects were fed known quantities of the target nut or nut oil in a nutrient defined diet. The studies have varied with respect to length, number of treatments, 'dose' of nut/nut oil, as well as the endpoints measured. Typically, the studies have been designed to test the hypothesis that the test diet with nuts elicits a favourable lipid and lipoprotein response compared with a Western diet and/or a lower fat diet that is recommended for cholesterol lowering.

The studies to date have consistently shown that a nut/nut oil-containing diet, low in saturated fat and cholesterol, beneficially affects lipids and lipoproteins compared to the control diet (typically either a low fat diet or an average American/western diet). (Table 1) The favourable effects of the nut/nut oil diet on the plasma lipid and lipoprotein profile is a mechanism that appears to account for some of the cardioprotective effects observed in the epidemiologic studies. The purpose of this review is to examine the evidence on the effects of tree nuts on established lipid and lipoprotein risk factors, including total cholesterol (TC), LDL cholesterol (LDL-C), HDL cholesterol (HDL-C), and triglycerides (TG). In addition, the effects of nut consumption on more contemporary lipid/lipoprotein risk factors including non-HDL cholesterol and the ratios of TC:HDL-C and LDL-C:HDL-C will be reviewed. Since oxidative stress is a key event in atherogenesis, and many studies have evaluated the effect of nut consumption on LDL oxidation, this review will also examine this literature. As will become evident, there is an impressive database showing that tree nut consumption improves the lipid $\mathrm{CHD}$ risk factor profile, which may explain the cardio-protective effects of nut consumption that have been demonstrated in the epidemiologic studies.

Nuts have a unique nutrient profile that beneficially affects lipids and lipoproteins. This is due largely to the favourable fatty acid profile of nuts. Nuts are low in saturated fatty acids and high in unsaturated fatty acids, including monounsaturated fatty acids, omega- 6 and omega- 3 fatty acids. In addition, nuts are a source of dietary fibre; soluble fibre has a blood cholesterol-lowering effect. Collectively, the fatty acid profile and fibre content of nuts can markedly lower LDL cholesterol. In fact, the cholesterol reduction observed in clinical studies of nuts is approximately $25 \%$ greater than would be expected based on blood cholesterol-predictive equations (Table 2) (Kris-Etherton et al. 1999). Ten out of seventeen controlled feeding studies in this review demonstrate a decrease in LDL-C that is greater than that which would be predicted using blood cholesterol-predictive 


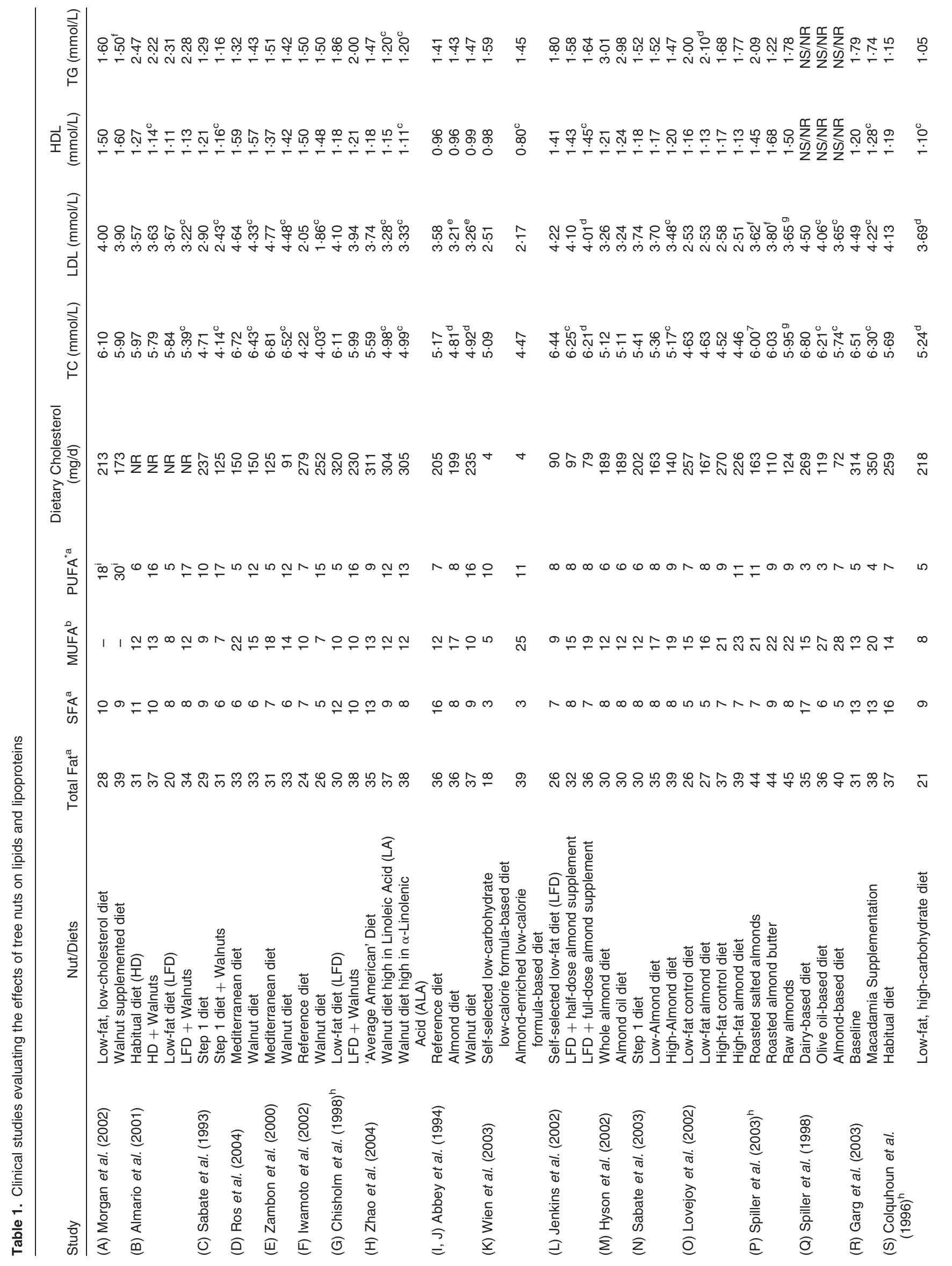




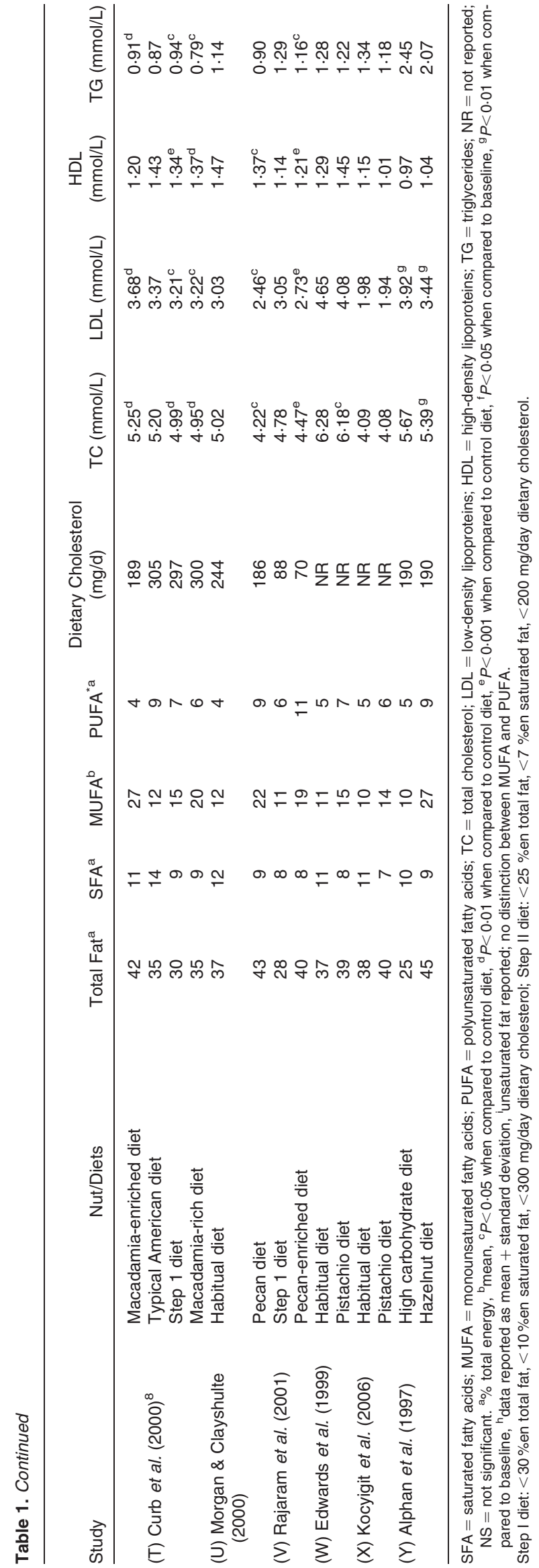

equations (Fig. 1). The predicted average decrease in LDL-C for these seventeen studies is $-0.23 \mathrm{mmol} / \mathrm{L}$, with an observed decrease of $-0.29 \mathrm{mmol} / \mathrm{L}$, when comparing the tree nut-rich diet to the control diet. It has been speculated that other nut constituents, such as plant protein, and possibly other factors (that remain to be identified) could account for this effect. Other nutrients in nuts that favourably affect lipids and lipoproteins are antioxidants, including vitamin E, vitamins and minerals, such as folate, potassium, magnesium, as well as non-nutrient bioactive compounds, such as phytosterols and other phytochemical compounds including ellagic acid, flavonoids, phenolic compounds, luteolin, and tocotrienols (Kris-Etherton et al. 2001) (Table 3). Many of these could protect LDL against oxidative modification, others reduce cholesterol absorption and thereby decrease total and LDL-cholesterol levels. Thus, the nutrient profile of nuts favourably affects lipid and lipoprotein CVD risk factors in a diversity of ways.

\section{Walnuts}

The effects that walnuts have on blood lipid and lipoprotein reduction have been studied extensively. While most tree nuts are rich in monounsaturated fatty acids (MUFA), walnuts are unique in that they contain high levels of polyunsaturated fatty acids (PUFA), both $\alpha$-linolenic acid and linoleic acid (Feldman, 2002). Walnuts are also high in several cardio-protective nutrients including fibre, vitamin $\mathrm{E}$, folate and potassium. Studies evaluating the effects of walnuts on blood lipids and lipoproteins have been conducted in both controlled feeding and free-living settings in healthy individuals and those with elevated cholesterol levels, generally as part of a cholesterol-lowering diet.

\section{Supplementation studies}

Several investigators have tested the effect of walnut supplementation on blood lipids and lipoproteins. In a study conducted by Chisholm et al. (1998), a free-living two-period randomized crossover design was used to test whether or not a $78 \mathrm{~g} / \mathrm{d}$ supplement of walnuts would have added effects on the reduction of blood lipids, beyond that of a fat-modified (30\% totaSl fat) diet. The walnut supplementation represented $20 \%$ of the total energy in the supplemented diet and accounted for $55 \%$ of the total fat $(38 \%)$ in the diet. The reductions observed in LDL-cholesterol $(-8 \%)$, total cholesterol $(-4 \%)$, and apolipoprotein B $(-13 \%)$ were statistically significant $(P<0.01)$ following the walnut supplemented diet, when compared to baseline. While the low-fat diet elicited similar responses on LDL-cholesterol $(-5 \%)$, total cholesterol $(-2 \%)$, and apolipoprotein $\mathrm{B}(-8 \%)$, these results were not significantly different from baseline levels. The increases observed in HDL-cholesterol following the low-fat $(11 \%)$ and walnut-supplemented (14\%) diets were both significantly different from baseline levels, however they were not different from one another. These results indicate that incorporating walnuts $(78 \mathrm{~g} / \mathrm{d})$ in the diet enhances the lipid and lipoprotein response to a blood cholesterol-lowering diet.

Almario et al. (2001) tested the effect of walnut supplementation in hypercholesterolemic men and women on both a fatmodified and a habitual diet. Subjects consumed the following 
Tree nuts and the lipid profile: a review of clinical studies

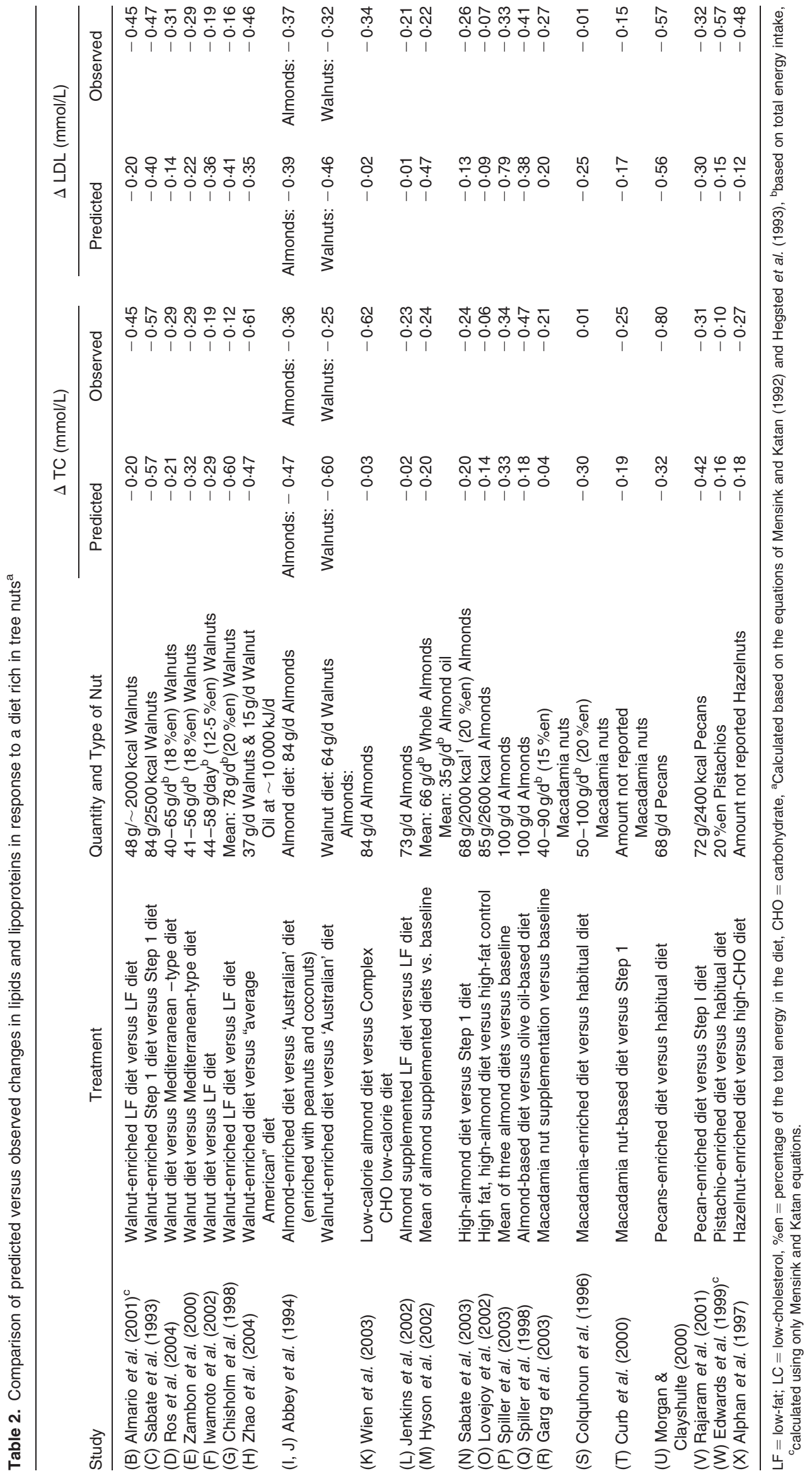




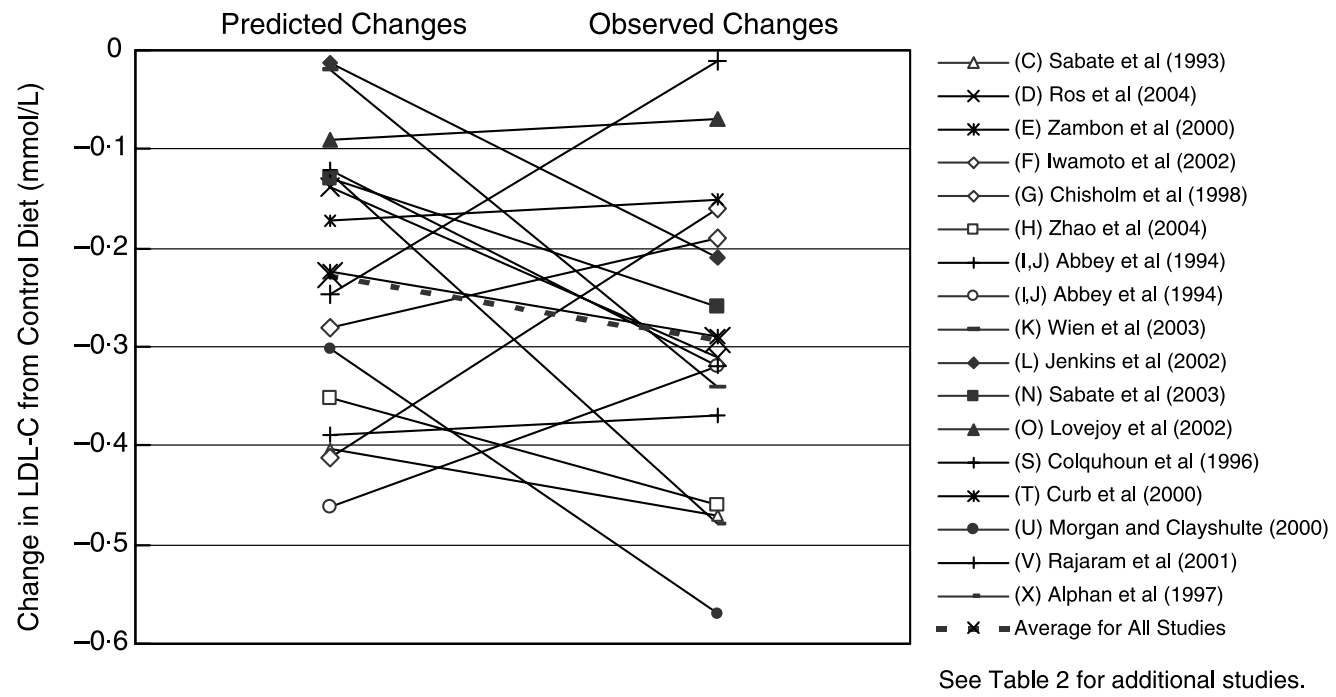

Fig. 1. Predicted $v$. observed changes in LDL-C in tree nut studies.

Table 3. Nutrients for the reduction of lipids and lipoproteins (per $100 \mathrm{~g} \mathrm{nuts}^{\mathrm{a}}$ )

\begin{tabular}{|c|c|c|c|c|c|c|c|c|c|c|}
\hline Nut & $\begin{array}{l}\text { SFA } \\
(\mathrm{g})\end{array}$ & $\begin{array}{l}\text { MUFA } \\
(\mathrm{g})\end{array}$ & $\begin{array}{l}\text { PUFA } \\
\text { (g) }\end{array}$ & $\begin{array}{l}\text { Linoleic Acid } \\
\text { (g) }\end{array}$ & $\begin{array}{l}\text { Linolenic Acid } \\
\text { (g) }\end{array}$ & $\begin{array}{l}\text { Cholesterol } \\
\text { (mg) }\end{array}$ & $\begin{array}{l}\text { Fiber } \\
(\mathrm{g})\end{array}$ & $\begin{array}{l}\alpha \text {-tocopherols } \\
\text { (mg) }\end{array}$ & $\begin{array}{l}\text { Total Phytosterols } \\
\text { (mg) }\end{array}$ & $\begin{array}{l}\text { B-Sitosterol } \\
\text { (mg) }\end{array}$ \\
\hline Walnuts & 6 & 9 & 47 & 38 & 9 & 0 & 7 & 0.70 & 72 & 64 \\
\hline Almonds & 4 & 32 & 12 & 12 & 0 & 0 & 12 & 25.87 & 120 & 111 \\
\hline Macadamias & 12 & 59 & 1.5 & 1.30 & 0.20 & 0 & 8 & 0.57 & 114 & 107 \\
\hline Pecans & 6 & 41 & 22 & 21 & 1 & 0 & 10 & 1.4 & 102 & 89 \\
\hline Pistachios & 6 & 24 & 14 & 13.64 & 0.26 & 0 & 10 & 1.93 & 214 & 199 \\
\hline Hazelnuts & 4 & 46 & 8 & 7.83 & 0.09 & 0 & 10 & 15.03 & 96 & 89 \\
\hline Brazils & 16 & 23 & 24 & 23.81 & 0.06 & 0 & 5 & $\mathrm{n} / \mathrm{a}$ & $\mathrm{n} / \mathrm{a}$ & $\mathrm{n} / \mathrm{a}$ \\
\hline Cashews & 9 & 27 & 8 & 7.66 & 0.16 & 0 & 3 & $\mathrm{n} / \mathrm{a}$ & 158 & $\mathrm{n} / \mathrm{a}$ \\
\hline Pine nuts & 8 & 19 & 21 & 21 & 1 & 0 & 4 & $\mathrm{n} / \mathrm{a}$ & 141 & $n / a$ \\
\hline
\end{tabular}

Source: USDA National Nutrient Database for Standard Reference, Release 15, August 2002.

${ }^{a}$ All of the nuts are unsalted; almonds, brazilnuts, hazelnuts, pecans, pine nuts and walnuts are unroasted; cashews, macadamias, and pistachios are dry roasted; SFA $=$ saturated fatty acids, MUFA = monounsaturated fatty acids, PUFA = polyunsaturated fatty acids.

diets in a sequential order: 1 ) habitual diet (HD; $31 \%$ total fat, $11 \%$ SFA, $12 \%$ MUFA, $6 \%$ PUFA); 2) habitual diet + walnuts (HDW; $37 \%$ total fat, $10 \%$ SFA, $13 \%$ MUFA, $16 \%$ PUFA); 3 ) low-fat diet (LFD; $20 \%$ total fat, $8 \%$ SFA, $8 \%$ MUFA, 5\% PUFA); and 4) low-fat diet + walnuts (LFDW; $34 \%$ total fat, $8 \%$ SFA, $12 \%$ MUFA, $17 \%$ PUFA). The walnut dosage was $48 \mathrm{~g}$ per $2020 \mathrm{kcal}$ for each of the supplemented diets. While the addition of walnuts to the habitual diet elicited a $3 \%$ reduction in total cholesterol and a $10 \%$ reduction in triglycerides, there was also a non-significant $2 \%$ increase in LDL-cholesterol and a $10 \%$ decrease in HDL-cholesterol. The HDL response to the walnut supplementation was the only significant effect $(P<0 \cdot 01)$. On the contrary, the supplementation of the same dosage of walnuts to a low-fat, low saturated fat diet resulted in significant reductions $(P<0.01)$ in both total $(8 \%)$ and LDL-cholesterol $(12 \%)$, accompanied by insignificant changes in triglycerides $(-1 \%)$ and HDL-cholesterol $(+2 \%)$. In addition to the standard lipid profile, the investigators also measured the lipoprotein subclasses and particle sizes. One of the emerging risk factors for cardiovascular disease is the presence of small, dense LDL particles. These smaller LDL particles are formed primarily in response to an elevation in triglycerides and are associated with an increased risk for coronary heart disease (Austin et al. 1990). Results of this study demonstrate that walnut supplementation lowers cholesterol preferentially in the small, dense LDL particles. The addition of walnuts to the HD decreased the number of small dense LDL ( $46 \%$ on HD v. $33 \%$ on HDW; $P<0 \cdot 01)$. Consequently, the lowfat diet attenuated this change $(42 \%$ of the particles were small dense LDL), and the addition of walnuts to the lowfat diet had no effect (39\% of the particles were small dense LDL). These results indicate that walnut supplementation may create a favourable shift among the lipoprotein distributions even when total LDL-C levels remain unchanged.

\section{Studies that incorporate walnuts in the diet}

Walnuts have also been used to elicit a reduction in blood lipids and lipoproteins when they are incorporated into a diet plan. A recent controlled clinical feeding trial evaluated the effects of two walnut-rich diets on risk factors for CVD (Zhao et al. 2004). Hypercholesterolemic subjects $(n=23)$ consumed each diet for 6 weeks in a randomized, threeperiod crossover design: 1) Average American diet (AAD); [34\% total fat, $13 \%$ SFA, $13 \%$ MUFA, 9\% PUFA $(7.7 \%$ 
LA, 0.8\% ALA)], 2) Linoleic Acid diet (LA); [37\% total fat, $9 \%$ SFA, $12 \%$ MUFA, $16 \%$ PUFA (12.6\% LA, $3.6 \%$ ALA)], and 3) $\alpha$-Linolenic Acid diet (ALA); [38\% total fat, $8 \%$ SFA, $12 \%$ MUFA, $17 \%$ PUFA (10.5\% LA, $6.5 \%$ ALA)]. Walnuts and walnut oil represented one-half of the total fat in the two high-PUFA diets. The daily consumption of walnuts and walnut oil was 37 and $15 \mathrm{~g}$, respectively, for a diet that provided $2400 \mathrm{kcal} / \mathrm{d}$. Flaxseed oil, $\sim 20 \mathrm{~g} / \mathrm{d}$ for an energy intake of $2400 \mathrm{kcal} / \mathrm{d}$, was also used to increase the ALA content of the ALA diet. Compared with the AAD, the two high-PUFA diets elicited similar lipid-lowering responses. Serum TC, LDL-C and TG were 10.9, 12.3 and $18.4 \%$ lower, respectively, following the consumption of the LA diet $(P<0.05)$, and were $10.8,11.0$ and $18.4 \%$ lower, respectively, when comparing the ALA diet to the AAD $(P<0 \cdot 05)$. Despite a significant decrease in HDL-C following the ALA diet $(P<0.05)$, compared to the AAD, there were no differences in HDL-C response following the LA and ALA diets; the two high-PUFA diets reduced the ratio of TC:HDL-C similarly. Iwamoto et al. (2002) demonstrated analogous improvements in the lipid profile with the incorporation of $44-58 \mathrm{~g}$ of walnuts per day $(12.5 \% \mathrm{en})$ into a low-fat $(25 \%$ total fat) diet. Significant reductions $(P<0.001)$ in both total $(-4.5 \%)$ and LDL-cholesterol $(-9.8 \%)$ were observed when comparing the walnut diet to the low-fat diet. There were no changes in HDL-cholesterol and triglycerides as a result of the incorporation of walnuts into the diet.

Results from the Barcelona Walnut Trial (Zambon et al. 2000) illustrated the potent lipid lowering effect of walnuts, compared to a Mediterranean diet, high in monounsaturated fatty acids. Despite a higher amount of total fat (33 v. 30\% in a Mediterranean diet) and an equal amount of saturated fat in both diets (5\%) the walnut diet $(41-56 \mathrm{~g} / \mathrm{d} ; 18 \%$ en) elicited a $9 \%$ reduction in total cholesterol and an $11 \%$ reduction in LDL-cholesterol when compared to the Mediterranean diet. In addition, despite an enrichment of the LDLcholesterol with polyunsaturated fatty acids while on the walnut diet, the resistance of these LDL particles to oxidation was preserved. In a similar study, when compared to a low-fat, low-cholesterol diet ( $28 \%$ total fat), the incorporation of $64 \mathrm{~g} / \mathrm{d}$ of walnuts into a cholesterol-lowering diet $(39 \%$ total fat) resulted in a significant reduction $(P<0.05)$ in serum triglycerides $(15 \%)$, with a trend for a further improvement in the lipid profile, as indicated by a reduction in total $(3 \%)$ and LDL-cholesterol (3\%) and an increase in HDL-cholesterol (2\%) (Morgan et al. 2002). Even greater improvements have been observed with the incorporation of $84 \mathrm{~g}$ of walnuts per $2500 \mathrm{kcal}$ to a Step 1 diet (Sabate et al. 1993). As a result of this randomized 4-week crossover study, the incorporation of walnuts into a Step 1 diet resulted in a significant improvement in the lipid profile, characterized by a reduction in total cholesterol $(-12 \% ; P<0.001)$, LDL-cholesterol $(-16 \%$; $P<0.001)$ and the ratios of total:HDL-cholesterol (4.0 to $3.7 ; \quad P<0.001)$ and LDL:HDL-cholesterol $(2.5$ to 2.2 ; $P<0.001)$. These results were also accompanied by an $8 \%$ reduction in triglycerides and a $5 \%$ reduction in HDL-cholesterol $(P<0 \cdot 01)$.

Ros and colleagues (2004) tested the hypothesis that nuts, walnuts in particular, would not only improve hypercholesterolemia, but would also favourably affect endothelial function in subjects with hypercholesterolemia. In addition to hypercholesterolemia, endothelial dysfunction is associated with atherosclerosis and the development of vascular disease. Because nuts proportionally contain a large amount of arginine, the precursor of the endogenous vasodilator nitric oxide, this would indicate that nuts could also have a marked effect on cardiovascular health via an improvement in endothelial function. They conducted a two-period randomized crossover design feeding study in twenty-one hypercholesterolemic men and women. The diets included in the design were a Mediterranean-type cholesterol-lowering diet and a walnut-enriched diet, both of which contained $33 \%$ of calories from total fat. The walnut-enriched diet was developed by substituting $\sim 32 \%$ of the calories from MUFA in the cholesterol-lowering Mediterranean diet. As a result of the study, the walnut-enriched diet elicited significant reductions in total $(-4.4 \% ; P<0.05)$ and LDL-cholesterol $(-6.4 \% ; P=0.01)$ compared to the Mediterranean diet. In addition to the favourable effects on the lipid profile, the walnut-enriched diet also led to a $64 \%$ increase in endothelium-dependent vasodilation. The results of this study therefore help to explain the additional cardio-protective effects of walnuts, beyond simply the reduction of blood cholesterol levels.

\begin{abstract}
Almonds
Numerous studies with almonds have been conducted to assess their effects on lipids and lipoproteins. Because almonds are a rich source of monounsaturated fatty acids, and low in saturated fat, they can be used as a fat source to achieve a moderate fat diet that is low in SFA. Almonds contain a significant amount of $\alpha$-tocopherol, a potent antioxidant, and many other proposed cardio-protective components, including folic acid, calcium, potassium, magnesium, copper, zinc and phytochemicals.
\end{abstract}

\section{Studies that evaluate almonds (raw and roasted almonds, almond butter and almond oil)}

Clinical trials conducted in healthy individuals, and individuals with high cholesterol and diabetes, have illustrated the cholesterol-lowering effect of almonds in both controlled and free-living situations. Some studies have assessed whether the processing of almonds has an effect on the lipid-lowering effect. These studies provide important information about whether processing almonds increases or decreases bioactive effects. Comparing whole almonds $v$. almond oil provides information about whether effects are due specifically to the oil fraction or whether there are benefits of both the fat and components of the total nutrient package. Hyson and colleagues (2002) used a two-period 6-week randomized crossover design to test whether the incorporation of whole almonds (66g) $v$. almond oil (35g) into a habitual diet would have different effects on blood lipids and lipoproteins. The goal of their study was to replace $50 \%$ of the subjects' usual total fat intake $(30 \%)$ with either whole almonds or almond oil. The only difference in the composition of the diets was a $36 \%$ increase in fibre during the whole almond diet, compared to both baseline and the almond oil diet. Both the whole almond and the almond oil diets significantly $(P<0.05)$ reduced total $(4 \%, 4 \%)$ and LDL-cholesterol $(6 \%$, 
$7 \%)$, triglycerides $(14 \%, 15 \%)$ and increased HDL-cholesterol $(4 \%, 7 \%)$, respectively, when compared to baseline. The results of this study indicate that the lipid-lowering effect of almonds is due primarily to the constituents in the lipid fraction of almonds. A similar study tested the effects of incorporating either: 1) $100 \mathrm{~g}$ roasted salted almonds; 2) $100 \mathrm{~g}$ roasted almond butter; or 3) $100 \mathrm{~g}$ raw almonds into a cholesterol-lowering diet (Spiller et al. 2003). Each diet contained $\sim 44 \%$ total fat, $\sim 8 \%$ SFA, $\sim 22 \%$ MUFA, $\sim 10 \%$ PUFA. As a result of the study there was a significant reduction $(P<0 \cdot 05)$ in LDL-C from all forms of almonds (12\% for raw almonds and $7 \%$ for roasted almonds and almond butter). The reduction in total cholesterol also was greatest following the raw almond diet $(7 \% ; P<0.01)$ and was similar for the roasted almond $(5 \% ; P<0.05)$ and almond butter diets $(5 \%)$. There were no significant changes in HDL-cholesterol or triglycerides following the three different diets.

\section{Studies that incorporate almonds in the diet}

In addition to testing the different forms of almonds, researchers also have evaluated the effects of incorporating almonds into a lipid-lowering diet. Spiller and colleagues (1998) evaluated the lipid-lowering effects of an almond-based diet when compared to an olive oil-based diet and a dairy-based control diet. Forty-five hypercholesterolemic males and females were randomized to receive one of the three treatments in this 4-week parallel design study. The study diets were as follows: 1) almond-based diet (40\% total fat, 5\% SFA, $28 \%$ MUFA, $7 \%$ PUFA); 2 ) olive oil-based diet (36\% total fat, $6 \%$ SFA, $27 \%$ MUFA, 3\% PUFA); and 3) dairy-based diet (35\% total fat, $17 \%$ SFA, $15 \%$ MUFA, $3 \%$ PUFA). The almondbased diet (higher in total fat) produced the greatest reductions in total (16\%) and LDL-cholesterol (19\%), when compared to the dairy-based diet $(P<0.001)$. The reductions in total $(9 \%)$ and LDL-cholesterol $(10 \%)$ also were statistically significant $(P<0.001)$ when comparing the olive-oil based diet to the dairy-based diet. There were no significant changes for HDL-cholesterol or triglycerides across all three diets.

In a 24-week free-living weight loss trial, Wien and colleagues (2003) evaluated the effect of incorporating $84 \mathrm{~g} /$ day of almonds into a formula-based low-calorie diet (LCD) (39\% total fat, $3 \%$ SFA, $25 \%$ MUFA, $11 \%$ PUFA) in comparison to a complex-carbohydrate formula-based LCD (18\% total fat, $3 \%$ SFA, $5 \%$ MUFA, $10 \%$ PUFA). Improvements were observed in the lipid profile, indicated by reductions in total $(13 \%, 9 \%)$, LDL-cholesterol $(15 \%, 10 \%)$ and triglycerides $(29 \%, 27 \%)$, following the almond-enriched diet and the complex carbohydrate diet, respectively. None of these changes were different between the two diet treatments. The changes observed in HDL-cholesterol were statistically different $(P<0 \cdot 05)$ between the two diets, with a $6 \%$ reduction in HDL-cholesterol following the almond-enriched diet and a $15 \%$ increase following the complex-carbohydrate diet. While both groups did lose weight, the loss was greater $(P<0.0001)$ for the almond-enriched diet $(18 \%)$, compared to the complex-carbohydrate diet $(11 \%)$. Overall, the almond-enriched LCD produced a sustained and greater weight loss in comparison to the complex-carbohydrate LCD, accompanied by improvements in the lipid profile.
Researchers have also studied the effects of the incorporation of almonds into diets with a greater variation in the levels of total fat in individuals with type 2 diabetes (Lovejoy et al. 2002). A 4-week, four-period randomized crossover study design was used to evaluate the lipid-lowering effect of the incorporation of walnuts into both a high-fat and a low-fat diet. The following four diets were used: 1) low-fat control (LFC; $25 \%$ total fat, $10 \%$ from olive or canola oil); 2) low-fat, high-almond (LFA; $25 \%$ total fat, $10 \%$ from almonds); 3) high-fat control (HFC; $37 \%$ total fat, $10 \%$ from olive or canola oil); 4) high-fat, high-almond (HFA; $37 \%$ total fat, $10 \%$ from almonds). Total cholesterol was the lowest following the HFA diet $(4.46,4.52,4.63$, $4.63 \mathrm{mmol} / \mathrm{L}$ for the HFA, HFC, LFA, and LFC diets, respectively; $P<0.001)$. There was a similar effect from adding almonds to either a high fat or low fat diet compared with the high fat, low fat control diets on triglycerides. However the high-fat diets elicited lower triglyceride levels when compared to the low-fat diets $(1.77,1.68,2.10,2.0 \mathrm{mmol} / \mathrm{L}$ for the HFA, HFC, LFA, and LFC diets, respectively; $P<0.001$ ). The only main effect of fat source (almonds $v$. olive and canola oils) was found for HDL-cholesterol, with the two almond diets producing the lowest levels of HDLcholesterol $(1.13,1.17,1.13,1.16 \mathrm{mmol} / \mathrm{L}$ for the HFA, HFC, LFA, and LFC diets, respectively; $P<0 \cdot 01)$. There was no significant effect from fat level or fat source on LDL-cholesterol, however there was a trend for an effect of fat source $(2.51,2.58,2.53,2.53 \mathrm{mmol} / \mathrm{L}$ for the HFA, HFC, LFA, and LFC diets, respectively; $P=0 \cdot 06$ ). There was no significant effect of fat source on LDL:HDL-cholesterol ratio.

\section{Dose-response studies}

A dose-response relationship was observed with the incorporation of almonds into a Step II diet (Jenkins et al. 2002). Subjects consumed a Step II diet plus either: 1) $73 \mathrm{~g} / \mathrm{d}$ of whole almonds; 2) muffins; or 3 ) half portions of almonds ( $37 \mathrm{~g} / \mathrm{d}$ ) and muffins, in a randomized, crossover design study. Each supplement provided $22.2 \%$ of the total energy in the diet. Again, a dose-response relationship was observed; the full portion of almonds was associated with a $5.6 \%$ reduction in TC, a $9.4 \%$ reduction in LDL-C, an $8.4 \%$ reduction in the ratio of TC:HDL-C, and a $3.8 \%$ increase in HDL-C, compared to baseline. These improvements in the lipid profile translate into a $9.2 \%$ reduction in CHD risk following the consumption of $73 \mathrm{~g}$ of walnuts per day.

Using a randomized crossover design, Sabate et al. (2003) conducted a dose-response study to test the effects of two different levels of almond intake compared with the NCEP Step I diet. Twenty-five healthy individuals consumed the following three isoenergetic diets for 4 weeks following a 2-week run-in diet: Step I diet (30\% total fat), low-almond diet (35\% total fat), high-almond diet (39\% total fat). Almonds represented $0 \%, 10 \%(\sim 34 \mathrm{~g} / 2000 \mathrm{kcal})$ and $20 \%$ ( $\sim 68 \mathrm{~g} / 2000 \mathrm{kcal})$ during the Step I, low-almond and high-almond diets, respectively. Levels of TC, LDL-C, apolipoprotein $\mathrm{B}$, and the LDL-C:HDL-C ratio were reduced in a dose-response manner (TC: $5.41,5.36,5.17 \mathrm{mmol} / \mathrm{L}$ and LDL-C $3.74,3.70,3.48 \mathrm{mmol} / \mathrm{L}$, respectively for Step-1, low-almond and high-almond diets). There were no significant differences in levels of HDL-cholesterol or triglycerides. 
This study suggests that the incorporation of almonds into a Step I diet will provide additional improvements to the lipid and lipoprotein profile, above those seen with the traditional Step I diet.

\section{Combination of almonds and walnuts}

In a study conducted by Abbey et al. (1994), the lipid-lowering effect of both almonds and walnuts were evaluated. Individuals consumed the following three diets for 3 weeks each in the following order: 1) Reference diet (enriched with peanuts and coconuts; $36 \%$ total fat, $16 \%$ SFA, $12 \%$ MUFA, $7 \%$ PUFA); 2) Almond-enriched diet $(84 \mathrm{~g} / \mathrm{d} ; 36 \%$ total fat, $8 \%$ SFA, $17 \%$ MUFA, $8 \%$ PUFA); and 3) Walnut-enriched diet (68 g/day; $37 \%$ total fat, 9\% SFA, $10 \%$ MUFA, $16 \%$ PUFA). Both doses of nuts were equivalent to $46 \mathrm{~g}$ of fat. Individuals were instructed on how to incorporate these fat sources (i.e. peanuts, coconut, almonds and walnuts) into their diets. Compared to the reference diet, there were significant reductions in TC and LDLC, $7 \%(P<0.01)$ and $10 \%(P<0.001)$, respectively, after supplementation with almonds, and $5 \%(P<0.01)$ and $9 \%$ $(P<0.001)$, respectively, after supplementation with walnuts. There were no significant changes in triglyceride and HDL-C levels across the three diets.

\section{Macadamia nuts}

Macadamia nuts, a rich source of monounsaturated fatty acids, are typically eaten as a snack and used in confectionary products and for the elaboration of chocolate. To date, three clinical trials have evaluated the effect of macadamia nuts on the lipid and lipoprotein profile. These studies have also used macadamia nuts as a tool to reduce the saturated fat in the diet by replacing it with monounsaturated fats from the macadamia nuts. The results of a supplement trial by Garg et al. (2003) indicate that the addition of macadamia nuts, representing $15 \%$ of the total energy intake $(40-90 \mathrm{~g} / \mathrm{d})$, results in a significant decrease in TC $(3.0 \%)$ and LDL-C $(5.3 \%)$, and a concurrent increase in HDL-C $(7.9 \%)$ in hypercholesterolemic men.

Two controlled feeding trials have been conducted and have likewise demonstrated an improvement of the lipid and lipoprotein profile with the incorporation of macadamia nuts. In the first trial, researchers compared a high carbohydrate diet (HCD; $21 \%$ total fat) and a macadamia-enriched diet (MD; $42 \%$ total fat) to the subjects' typical food intake (37\% total fat) (Colquhoun et al. 1996). Both the HCD and MD elicited a significant reduction in TC $(-7.9 \%)$ and LDL-C $(-10.7 \%)$ when compared to the typical food intake. The MD produced further improvements, compared with the $\mathrm{HCD}$, in that it reduced triglyceride levels by $20.9 \%$. In addition, the HCD reduced HDL-C by $13.1 \%$, while the MD maintained HDL levels. In a later study, Curb et al. (2000) compared a macadamia nut based diet (37\% total fat) to a 'typical American' diet (37\% total fat) and a Step 1 diet (30\% total fat). When compared to the typical American diet, both the macadamia based diet and the Step 1 diet reduced TC (5\%, 4\%; $P<0.01)$, LDL-C (4\%, 5\%; $P<0.05)$, and HDL-C (4\%; $P<0.01,6 \% ; P<0.001)$, respectively. Triglyceride levels were higher on the Step 1 diet $(8 \%$; $P<0.05)$, when compared to the typical American diet, however the macadamia nut diet produced a significant reduction in triglyceride levels $(9 \% ; P<0.05)$.

\section{Pecans}

Pecans are a rich source of monounsaturated fatty acids, and a number of cardio-protective compounds, including plant sterols, vitamin E, folic acid, calcium, magnesium, phosphorus, zinc, vitamin $\mathrm{A}$ and several $\mathrm{B}$ vitamins. In addition, one serving of pecans provides approximately $10 \%$ of the daily value for both zinc and fibre.

Pecans have been used in two controlled feeding trials to assess their lipid-lowering effect in both healthy and hypercholesterolemic individuals. When compared to a self-selected diet, an 8-week supplement of $68 \mathrm{~g} / \mathrm{d}$ of pecans resulted in a $6 \%$ reduction in LDL cholesterol (Morgan \& Clayshulte, 2000). In addition, these normolipemic individuals also experienced a reduction in TC following pecan supplementation. In a later study, Rajaram et al. (2001) utilized a twoperiod crossover design to study the cholesterol lowering effect of pecans. Following a 2-week run-in phase of a Step I diet, subjects were then randomized to either continue with the Step I diet for the first 4-week period, or were placed on a pecan-enriched diet. While on the pecan-enriched diet, subjects consumed $72 \mathrm{~g}$ of pecans per $2400 \mathrm{kcal}$. When compared to the Step I diet, hypercholesterolemic individuals on the pecan-enriched diet experienced a $10.4 \%$ reduction in LDL cholesterol. Overall, the reduction observed on the pecanenriched diet compared to baseline $(-16 \%)$ represents a $25 \%$ reduction in CHD risk. In addition to a substantial reduction in LDL cholesterol, several other lipid and lipoprotein risk factors were reduced, including TC $(-6.7 \%)$, HDL cholesterol $(+5.6 \%)$, LDL:HDL $(-15.7 \%)$, TG $(-11.1 \%)$, apolipoprotein B $(-11.6 \%)$, and $\operatorname{Lp}(\mathrm{a})(-15.1 \%)$.

\section{Pistachio nuts}

Pistachio nuts are rich in monounsaturated fat and phytosterols, a plant sterol that has cholesterol-lowering properties. One serving ( $\sim 30 \mathrm{~g}$; approximately 49 nuts) of pistachios contains $13 \mathrm{~g}$ of total fat, of which only $1.5 \mathrm{~g}$ is saturated fat, and provides more than $10 \%$ of the daily value for dietary fibre, vitamin B-6, thiamin, phosphorus and copper and $61 \mathrm{mg}$ plant sterols.

To date, two studies have been conducted to test the cholesterol lowering ability of pistachio nuts. One study, conducted in hypercholesterolemic men and women, demonstrated that pistachio nuts decrease lipid and lipoprotein levels. Edwards et al. (1999) conducted a two-period, 3-week randomized crossover design study that investigated the effects of a $100 \mathrm{~g}$ (20\% en) supplementation of pistachio nuts to a typical diet. The composition of diets were as follows: 1) habitual diet (37\% total fat, $11 \%$ SFA, $11 \%$ MUFA, $5 \%$ PUFA); and 2) pistachio diet (39\% total fat, $8 \%$ SFA, $15 \%$ MUFA, $7 \%$ PUFA). When compared to a habitual diet, the pistachio-enriched diet reduced LDL cholesterol by $11 \%$. Although this reduction was not significant, there was a significant reduction in TC $(-3.7 \%)$, TC:HDL $(-14.6 \%)$ and LDL:HDL $(-9.4 \%)$. The substitution of pistachio nuts was used to alter the fatty acid profile of the diet through a reduction in SFA and an increase in MUFA and PUFA. In a similar study, the substitution of pistachio nuts for $20 \%$ of the daily caloric intake resulted in significant reductions in the ratios of TC:HDL-C and LDL-C:HDL-C $(P<0.001$ and $P<0.01$, respectively), and small non-significant reductions in triglycerides and LDL-C (Kocyigit et al., 2006). Given that 
pistachios contain the highest levels of plant sterols it is likely that additional mechanisms mediate their blood cholesterollowering effects.

\section{Hazelnuts}

Hazelnuts contain a number of proposed cardio-protective compounds, including fibre, vitamin E, arginine, folate, vitamin B-6, calcium, magnesium and potassium. In addition, hazelnuts are a rich source of monounsaturated fats. Hazelnuts contain approximately $91 \%$ monounsaturated fatty acids, mostly oleic acid, and less than $4 \%$ saturated fatty acids. This fatty acid profile of hazelnuts would be expected to reduce both total and LDL cholesterol levels when incorporated into a low saturated fat, low cholesterol diet. In a study conducted by Alphan and colleagues, the effects of hazelnuts on blood lipids and lipoproteins were evaluated in nineteen individuals with type 2 diabetes (Alphan et al. 1997). Individuals consumed a high-carbohydrate diet $(60 \%$ carbohydrate, $25 \%$ total fat, $10 \%$ SFA, $10 \%$ MUFA, $5 \%$ PUFA) for $30 \mathrm{~d}$, followed by a 15 -d washout period before consuming a hazelnut diet $(40 \%$ carbohydrate, $45 \%$ total fat, 9\% SFA, $27 \%$ MUFA, $9 \%$ PUFA) for $30 \mathrm{~d}$. LDL-cholesterol was significantly reduced $(P<0 \cdot 01)$ following both the hazelnut $(26 \%)$ and the high-carbohydrate $(16 \%)$ diets, when compared to baseline values. While both diets also reduced total cholesterol, this reduction was only significant $(P<0 \cdot 01)$ following the hazelnut diet $(12 \% ; 5 \%$ for high-carbohydrate diet). The changes observed in apolipoprotein $\mathrm{B}(+7 \%,-8 \%)$, HDLcholesterol $(+2 \%,+8 \%)$ and triglycerides $(-12 \%$, $-16 \%$ ) following the high-carbohydrate and hazelnut diets respectively, were not significantly different when compared to baseline. While this is the only published study on the effects of hazelnuts on blood lipids and lipoproteins, hazelnuts would be expected to have similar effects on lipids and lipoproteins compared to other tree nuts because they also are low in saturated fat and high in unsaturated fatty acids.

\section{Maximal cholesterol lowering achievable with a multicomponent diet}

Numerous clinical studies have demonstrated cholesterol-lowering effects of diets that contain nuts. For maximal total and LDL cholesterol reductions by diet, it is now evident that a total diet approach is necessary that emphasizes the lowest amount of saturated fat and cholesterol achievable as well as high levels of viscous fibre and plant sterols. A study conducted by Jenkins et al. $(2003,2005)$ showed that a vegetarian diet that was very low in saturated fat and cholesterol and high in plant sterols $(1 \mathrm{~g} / 1000$ calories $)$, soy protein $(21.4 \mathrm{~g} / 1000$ calories), viscous fibre $(9.8 \mathrm{~g} / 1000$ calories), and almonds (14 g/1000 calories) decreased LDL cholesterol by approximately $30 \%$, similar to that observed with low-dose statin therapy $(20 \mathrm{mg} / \mathrm{d})$. While this study used almonds, based on studies conducted to date with other tree nuts, it would be predicted that other nuts could be substituted to achieve the same blood cholesterol-lowering outcome. Thus, tree nuts can be an important part of a blood cholesterol-lowering diet that elicits a maximal effect attainable by diet that is comparable to low dose statin therapy.

\section{Beyond serum lipids}

We have transited a remarkable research era with tree nuts during which time many studies have shown beneficial effects of all tree nuts studied on lipids and lipoproteins, due in large part to their fatty acid profile (i.e. low in saturated fat and high in unsaturated fat, both MUFA and PUFA). Current dietary guidelines recommend inclusion of unsaturated fatty acids within the context of a nutritionally adequate diet that provides $20-35 \%$ of calories from total fat, with 5-10\% recommended for PUFA (USDA, 2005). There are exciting research findings about how PUFA exert their regulatory and metabolic effects on a host of biological systems, including decreasing triglyceride and fatty acid synthesis, and increasing mitochondrial $\beta$-oxidation, as well as peroxisome $\beta$-oxidation (Clarke, 2001). In addition, there is keen interest in understanding the role of PUFAs in oxidative stress and inflammation. Relative to the latter, the mechanisms by which LA and ALA affect inflammation need to be better understood. For example, there is recent evidence that LA, ALA as well as docosahexaenoic acid (DHA) decrease IL-6, IL-1 $\beta$, and TNF- $\alpha$ gene expression, and nuclear factor (NF)- $\kappa \mathrm{B}$ activation, whereas peroxisome proliferators-activated receptor- $\gamma$ (PPAR $\gamma$ ) DNA binding activity was increased (Zhao et al. 2005). These findings reinforce the need to learn more about the signal pathways that mediate the effects that PUFAs have on gene transcription in a variety of biological systems. Moreover, it will be important to clarify how individual PUFAs, including omega-6 and omega-3 fatty acids, regulate a variety of biological systems that affect chronic disease risk. In this regard, innovative controlled diet studies using tree nuts will be important to manipulate levels of individual PUFA to assess how different packages of PUFA affect metabolism and health outcomes.

We need to better understand how these regulatory mechanisms are altered in a way that seems to exacerbate the progression of CVD, metabolic syndrome and diabetes. While research is underway to resolve the mechanisms of PUFA action, we have a robust database on tree nuts that compellingly shows that they decrease the risk of CVD. It will be important to determine how different tree nuts included in a healthy diet affect a variety of biomarkers for CVD and other chronic diseases, and study the underlying mechanisms of action. It is abundantly clear that including food sources of PUFA, such as tree nuts, in the diet is an integral component of the new generation of healthy diets.

Beyond their fatty acid profile, tree nuts are a unique food because they are a rich source of dietary fibre, phytosterols and several micronutrients that have cardio-protective effects. The evidence base for health benefits of tree nuts is expanding beyond lipids and lipoproteins, and increasing to address how nuts affect metabolic syndrome and diabetes. There is a need to enlarge our understanding of the biological mechanisms that mediate the growing list of diverse metabolic effects of nut consumption. While unsaturated fatty acids in nuts are important for these health effects, it is clear that other bioactive compounds and nutrients in nuts play an important role. A better understanding of the 'nutrient package' in nuts may position us to develop innovative dietary strategies that confer health effects that extend beyond lipids and 


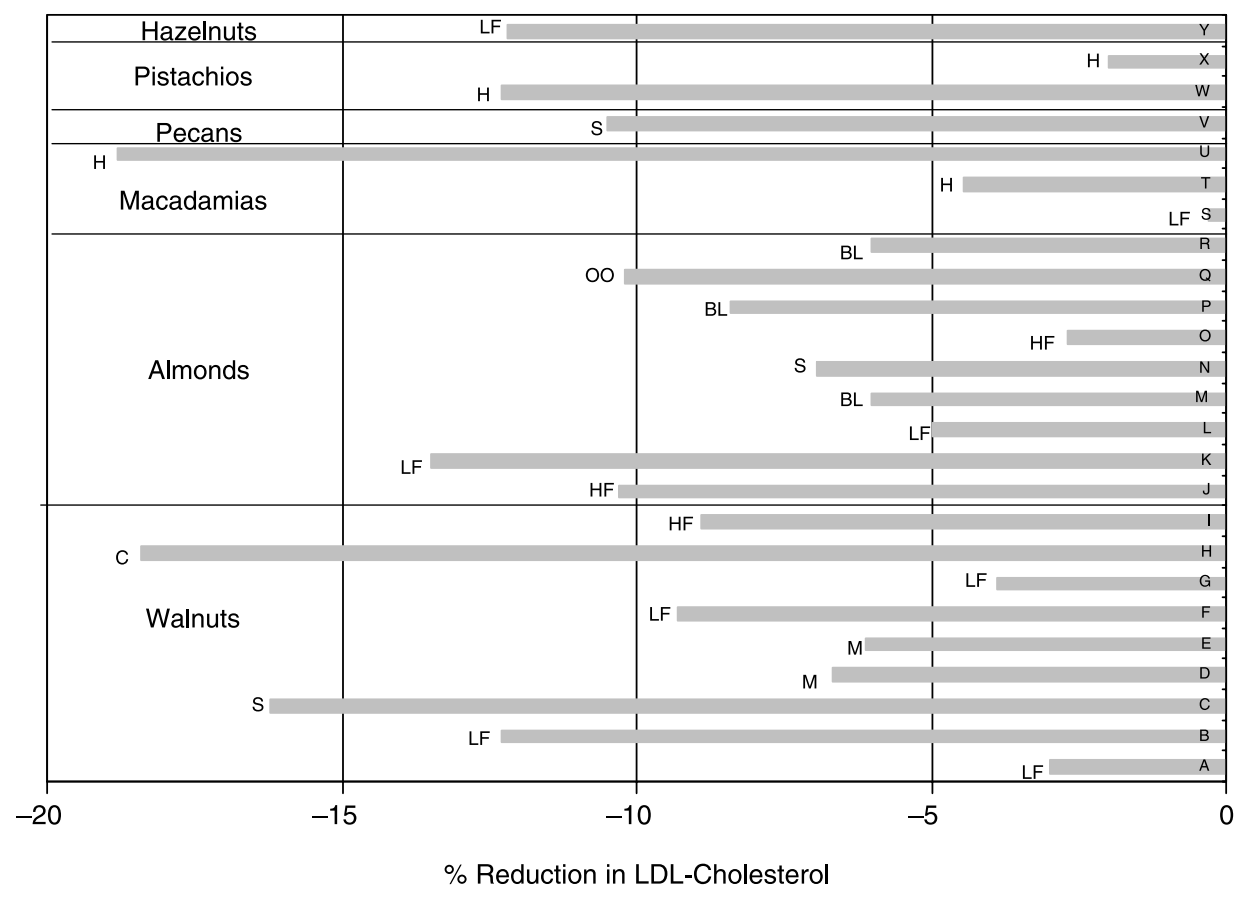

Note: Comparison reference diets at left of each column; $\mathrm{LF}=$ low-fat, $\mathrm{H}=$ habitual, $\mathrm{S}=\mathrm{Step} \mathrm{I}, \mathrm{BL}=\mathrm{Baseline}$, $\mathrm{OO}=$ olive oil-based diet, $\mathrm{HF}=$ high-fat, $\mathrm{M}=$ Mediterranean, $\mathrm{C}=$ Control; See Tables I and II for study details.

Fig. 2. LDL-Cholesterol lowering reported in tree nut studies.

lipoproteins and heart disease to other chronic diseases such as metabolic syndrome and diabetes.

\section{Conclusions}

The results of clinical trials with tree nuts have reported favourable effects on blood lipids and lipoproteins, major risk factors for CVD. Studies conducted to date have consistently demonstrated beneficial effects on lipids and lipoproteins, primarily a decrease in LDL-C (Fig. 2), which is predictive of a reduction in CHD risk. This effect has been demonstrated consistently in different population groups, utilizing various study designs and methods. A pooled analysis of fifteen clinical human nutrition studies that have evaluated the effect of tree nut containing diets on serum lipids and lipoproteins demonstrated a $0.34 \mathrm{mmol} / \mathrm{L}$ reduction in LDL-C when compared to a usual diet and a $0.23 \mathrm{mmol} / \mathrm{L}$ reduction when compared to a Step I Diet. The study designs included crossover (nine studies), parallel arm (three studies), and pre-post consecutive (three studies). The administration of diets and rigor of control varied from well-controlled metabolic type feeding studies (five studies), to dietary advice and dosage of nuts on freeliving individuals (three studies), to less rigid dietary advice and overall control (seven studies). With regard to the control of the diets, the well controlled feeding studies elicited a $14 \mathrm{mg} / \mathrm{dL}$ reduction in LDL-C, compared to only a $10 \mathrm{mg} / \mathrm{dL}$ reduction following the studies which were based on dietary advice and self-selected diets.

Tree nuts are a rich source of nutrients proven to reduce CHD risk through a reduction in lipid and lipoprotein risk factors. When nuts are incorporated into a healthy diet, there is an improvement in the lipoprotein profile. It is likely that future research could identify other bioactive compounds in nuts that confer additional health benefits beyond those identified to date.

\section{References}

Abbey M, Noakes M, Belling GB \& Nestel PJ (1994) Partial replacement of saturated fatty acids with almonds or walnuts lowers total plasma cholesterol and low-density-lipoprotein cholesterol. Am J Clin Nutr 59, 995-999.

Albert CM, Gaziano JM, Willett WC \& Manson JE (2002) Nut consumption and decreased risk of sudden cardiac death in the Physicians' Health Study. Arch Intern Med 162, 1382-1387.

Almario RU, Vonghavaravat V, Wong R \& Kasim-Karakas SE (2001) Effects of walnut consumption on plasma fatty acids and lipoproteins in combined hyperlipidemia. Am J Clin Nutr 74, 72-79.

Alphan EPM, Ackurt F \& Yilmaz T (1997) Nutritional composition of hazelnuts and its effects on glucose and lipid metabolism. In Proceedings of the fourth International Symposium on Hazelnut, Acta Hort, pp. 305-310 [AI Kosal and NT Gunes, editors].

Austin MA, King MC, Vranizan KM \& Krauss RM (1990) Atherogenic lipoprotein phenotype. A proposed genetic marker for coronary heart disease risk. Circulation 82, 495-506.

Chisholm A, Mann J, Skeaff M, Frampton C, Sutherland W, Duncan A \& Tiszavari S (1998) A diet rich in walnuts favourably influences plasma fatty acid profile in moderately hyperlipidaemic subjects. Eur J Clin Nutr 52, 12-16.

Clarke SD (2001) Polyunsaturated fatty acid regulation of gene transcription: a molecular mechanism to improve the metabolic syndrome. J Nutr 131, 1129-1132.

Colquhoun D, Humphries JA, Moores D \& Somerset SM (1996) Effects of a madacamia nut enriched diet on serum lipids and lipoproteins compared to a low fat diet. Food Australia 48, 216-222.

Curb JD, Wergowske G, Dobbs JC, Abbott RD \& Huang B (2000) Serum lipid effects of a high-monounsaturated fat diet based on macadamia nuts. Arch Intern Med 160, 1154-1158. 
Edwards K, Kwaw I, Matud J \& Kurtz I (1999) Effect of pistachio nuts on serum lipid levels in patients with moderate hypercholesterolemia. J Am Coll Nutr 18, 229-232.

Ellsworth JL, Kushi LH \& Folsom AR (2001) Frequent nut intake and risk of death from coronary heart disease and all causes in postmenopausal women: the Iowa Women's Health Study. Nutrition, metabolism, and cardiovascular diseases 11, 372-377.

Feldman EB (2002) The scientific evidence for a beneficial health relationship between walnuts and coronary heart disease. J Nutr 132, 1062S-1101S

Fraser GE, Sabate J, Beeson WL \& Strahan TM (1992) A possible protective effect of nut consumption on risk of coronary heart disease. The Adventist Health Study. Arch Intern Med 152, 1416-1424.

Fraser GE \& Shavlik DJ (1997) Risk factors for all-cause and coronary heart disease mortality in the oldest-old. The Adventist Health Study. Arch Intern Med 157, 2249-2258.

Fraser GE, Sumbureru D, Pribis P, Neil RL \& Frankson MA (1997) Association among health habits, risk factors, and all-cause mortality in a black California population. Epidemiology 8, 168-174.

Garg ML, Blake RJ \& Wills RB (2003) Macadamia nut consumption lowers plasma total and LDL cholesterol levels in hypercholesterolemic men. J Nutr 133, 1060-1063.

Hegsted DM, Ausman LM, Johnson JA \& Dallal GE (1993) Dietary fat and serum lipids: an evaluation of the experimental data. Am J Clin Nutr 57, 875-883.

Hu FB \& Stampfer MJ (1999) Nut consumption and risk of coronary heart disease: a review of epidemiologic evidence. Curr Atheroscler Rep 1, 204-209.

Hu FB, Stampfer MJ, Manson JE, Rimm EB, Colditz GA, Rosner BA, Speizer FE, Hennekens CH \& Willett WC (1998) Frequent nut consumption and risk of coronary heart disease in women: prospective cohort study. BMJ 317, 1341-1345.

Hyson DA, Schneeman BO \& Davis PA (2002) Almonds and almond oil have similar effects on plasma lipids and LDL oxidation in healthy men and women. J Nutr 132, 703-707.

Iwamoto M, Imaizumi K, Sato M, Hirooka Y, Sakai K, Takeshita A \& Kono M (2002) Serum lipid profiles in Japanese women and men during consumption of walnuts. Eur J Clin Nutr 56, 629-637.

Jenkins DJ, Kendall CW, Marchie A, Faulkner DA, Wong JM, de Souza R, Emam A, Parker TL, Vidgen E, Lapsley KG, Trautwein EA, Josse RG, Leiter LA \& Connelly PW (2003) Effects of a dietary portfolio of cholesterol-lowering foods vs lovastatin on serum lipids and C-reactive protein. JAMA 290, 502-510.

Jenkins DJ, Kendall CW, Marchie A, Faulkner DA, Wong JM, de Souza R, Emam A, Parker TL, Vidgen E, Trautwein EA, Lapsley KG, Josse RG, Leiter LA, Singer W \& Connelly PW (2005) Direct comparison of a dietary portfolio of cholesterol-lowering foods with a statin in hypercholesterolemic participants. Am J Clin Nutr 81, 380-387.

Jenkins DJ, Kendall CW, Marchie A, Parker TL, Connelly PW, Qian W, Haight JS, Faulkner D, Vidgen E, Lapsley KG \& Spiller GA (2002) Dose response of almonds on coronary heart disease risk factors: blood lipids, oxidized low-density lipoproteins, lipoprotein(a), homocysteine, and pulmonary nitric oxide: a randomized, controlled, crossover trial. Circulation 106, $1327-1332$.

Jiang R, Manson JE, Stampfer MJ, Liu S, Willett WC \& Hu FB (2002) Nut and peanut butter consumption and risk of type 2 diabetes in women. JAMA 288, 2554-2560.

Kocyigit A, Koylu AA, Keles H (2006) Effects of pistachio nuts consumption on plasma lipid profile and oxidative status in healthy volunteers. Nutr Metab Cardiovasc Dis 16, 202-209.

Kris-Etherton PM, Yu-Poth S, Sabate J, Ratcliffe HE, Zhao G \& Etherton TD (1999) Nuts and their bioactive constituents: effects on serum lipids and other factors that affect disease risk. Am J Clin Nutr 70, 504S-511S.

Kris-Etherton PM, Zhao G, Binkoski AE, Coval SM \& Etherton TD (2001) The effects of nuts on coronary heart disease risk. Nutr Rev 59, $103-111$.

Lovejoy JC, Most MM, Lefevre M, Greenway FL \& Rood JC (2002) Effect of diets enriched in almonds on insulin action and serum lipids in adults with normal glucose tolerance or type 2 diabetes. Am J Clin Nutr 76, 1000-1006.

Mensink RP \& Katan MB (1992) Effect of dietary fatty acids on serum lipids and lipoproteins. A meta-analysis of 27 trials. Arterioscler Thromb 12, 911-919.

Morgan JM, Horton K, Reese D, Carey C, Walker K \& Capuzzi DM (2002) Effects of walnut consumption as part of a low-fat, lowcholesterol diet on serum cardiovascular risk factors. Int $J$ Vitam Nutr Res 72, 341-347.

Morgan WA \& Clayshulte BJ (2000) Pecans lower low-density lipoprotein cholesterol in people with normal lipid levels. J Am Diet Assoc 100, 312-318.

Mukuddem-Petersen J, Oosthuizen W \& Jerling JC (2005) A systematic review of the effects of nuts on blood lipid profiles in humans. J Nutr 135, 2082-2089.

Rajaram S, Burke K, Connell B, Myint T \& Sabate J (2001) A monounsaturated fatty acid-rich pecan-enriched diet favorably alters the serum lipid profile of healthy men and women. J Nutr 131, 2275-2279.

Ros E, Nunez I, Perez-Heras A, Serra M, Gilabert R, Casals E \& Deulofeu R (2004) A walnut diet improves endothelial function in hypercholesterolemic subjects: a randomized crossover trial. Circulation 109, 1609-1614.

Sabate J, Fraser GE, Burke K, Knutsen SF, Bennett H \& Lindsted KD (1993) Effects of walnuts on serum lipid levels and blood pressure in normal men. $N$ Engl J Med 328, 603-607.

Sabate J, Haddad E, Tanzman JS, Jambazian P \& Rajaram S (2003) Serum lipid response to the graduated enrichment of a Step I diet with almonds: a randomized feeding trial. Am J Clin Nutr 77, 1379-1384.

Spiller GA, Jenkins DA, Bosello O, Gates JE, Cragen LN \& Bruce B (1998) Nuts and plasma lipids: an almond-based diet lowers LDL-C while preserving HDL-C. J Am Coll Nutr 17, 285-290.

Spiller GA, Miller A, Olivera K, Reynolds J, Miller B, Morse SJ, Dewell A \& Farquhar JW (2003) Effects of plant-based diets high in raw or roasted almonds, or roasted almond butter on serum lipoproteins in humans. J Am Coll Nutr 22, 195-200.

United States Department of Agriculture (USDA), United States Department of Health and Human Services (2005). Dietary Guidelines for Americans.

Wien MA, Sabate JM, Ikle DN, Cole SE \& Kandeel FR (2003) Almonds vs complex carbohydrates in a weight reduction program. Int J Obes Relat Metab Disord 27, 1365-1372.

Zambon D, Sabate J, Munoz S, Campero B, Casals E, Merlos M, Laguna JC \& Ros E (2000) Substituting walnuts for monounsaturated fat improves the serum lipid profile of hypercholesterolemic men and women. A randomized crossover trial. Ann Intern Med 132, 538-546.

Zhao G, Etherton TD, Martin KR, West SG, Gillies PJ \& Kris-Etherton PM (2004) Dietary alpha-linolenic acid reduces inflammatory and lipid cardiovascular risk factors in hypercholesterolemic men and women. J Nutr 134, 2991-2997.

Zhao G, Etherton TD, Martin KR, Vanden Heuvel JP, Gillies PJ, West SG \& Kris-Etherton PM (2005) Anti-inflammatory effects of polyunsaturated fatty acids in THP-1 cells. Biochem Biophys Res Comm 336, 909-917. 